\title{
Classificação morfológica na escala de detalhe no Pontal do Paranapanema, SP
}

\section{Morphological classification in detail scale in Pontal do Paranapanema, SP}

\author{
Anna Carolina dos Santos Cardoso* $\square$ (iD, Pablo Luíz Maia Nepomuceno $\square$ (iD \\ Departamento de Geografia, Faculdade de Filosofia, Letras e Ciências Humanas, \\ Universidade de São Paulo, São Paulo, Brasil \\ E-mail: pablo.nepomuceno@usp.br \\ *E-mail para correspondência: annacaroldsc@hotmail.com
}

Recebido (Received): 29/03/2019 Aceito (Accepted):30/07/2019

\begin{abstract}
Resumo: O presente artigo visa apresentar a viabilidade da classificação morfológica em escala de detalhe do relevo em bacias hidrográficas, de acordo com as legendas propostas por Colangelo (1996), correspondendo às Unidades Elementares do Relevo; e do quinto táxon da taxonomia proposta por Ross (1992), referente aos tipos e formas de vertentes com o uso de técnicas de geoprocessamento. Para tanto foi tomado como estudo de caso duas sub-bacias hidrográficas do Rio Santo Anastácio, localizadas na região do Pontal do Paranapanema. Os compartimentos morfológicos foram classificados através do processamento de Modelos Digitais de Elevação - MDE e ortofotos em ambiente de Sistemas de Informações Geográficas SIG. Estes dados foram disponibilizados pela Emplasa (2011), e são provenientes do projeto "Mapeia São Paulo", último levantamento sistemático de dados topográficos e aerofotogramétricos realizados para todo o território do estado de São Paulo. Como resultado foram obtidas as nove classes possíveis de Unidades Elementares do Relevo e, ao menos, seis classes diferentes de tipos e formas de vertentes, confirmando, portanto, a viabilidade técnica e metodológica dos procedimentos adotados no que se refere ao tratamento dos dados utilizados para a realização da classificação morfológica na escala de detalhe em bacias hidrográficas da região do Pontal do Paranapanema.
\end{abstract}

Palavras-chave: Mapeamento Morfológico; Modelos Digitais de Elevação; Morfologia de Vertentes; Geoprocessamento.

\begin{abstract}
The present article aims to present the feasibility of the morphological classification in a scale of relief detail in hydrographic basins, according to the legends proposed by Colangelo (1996), corresponding to the Elementary Units of Relief; and the fifth taxon of the taxonomy proposed by Ross (1992), referring to the types and forms of slopes using geoprocessing techniques. To this end, two hydrographic sub-basins of the Santo Anastácio River, located in the Pontal do Paranapanema region, were taken as a case study. The morphological compartments were classified through the processing of Digital Elevation Models - DEM and orthophoto in a Geographic Information Systems (GIS) environment. These data were made available by Emplasa (2011), and come from the "Mapeia São Paulo" project, the last systematic survey of topographic and aerophotogrammetric data carried out for the entire state of São Paulo. As a result, nine possible classes of Elementary Relief Units and, at least, six different classes of types and forms of slopes were obtained, thus confirming the technical and methodological feasibility of the procedures adopted about the treatment of the data used to carry out the classification. morphological in the detail scale in hydrographic basins of the Pontal do Paranapanema region.
\end{abstract}

Keywords: Morphological Mapping; Digital Elevation Models; Slope Morphology; Geographic Information Systems. 


\section{Introdução}

O mapeamento morfológico consiste em um dos estágios fundamentais do mapeamento geomorfológico, incorporando aspectos da morfografia, ou seja: do mapeamento qualitativo das formas que compõem um relevo; e da morfometria, ou seja: do mapeamento das características quantitativas do relevo (FLORENZANO, 2008; NEPOMUCENO; LUCHIARI, 2014), e por isso se faz também imprescindível ao mapeamento geotécnico e ao planejamento regional, ambiental e urbano.

Tais mapeamentos são tradicionalmente produzidos a partir da utilização de procedimentos de fotointerpretação estereoscópica, sobre fotografias aéreas verticais, articuladas com técnicas de obtenção de variáveis morfométricas por interpretação analógica de cartas topográficas. Entretanto, o intenso progresso nas técnicas de manipulação de dados topográficos por meio dos Sistemas de Informações Geográficas - SIG vem possibilitando novos meios para a obtenção de variáveis morfométricas, o que tem proporcionado uma ampliação da precisão e eficiência destes mapeamentos, bem como as suas possibilidades de aplicação (NEPOMUCENO; LUCHIARI, 2014).

Nesse sentido, Nepomuceno e Luchiari (2014) estabeleceram um conjunto de procedimentos técnicos e metodológicos de processamentos de Modelos Digitais de Elevação (MDEs) em ambiente SIG os quais apresentaram significativo sucesso na classificação morfológica de detalhe da área central do Município de Salesópolis-SP a partir de dados topográficos da Sabesp compatíveis até a escala 1:2.000 (NEPOMUCENO; LUCHIARI, 2014).

No entanto, ainda carece a realização de pesquisas acerca da viabilidade da aplicação destes procedimentos em áreas que apresentem características geomorfológicas distintas da utilizada por esses autores, assim como da viabilidade de utilização dos dados topográficos e aerofotogramétricos gerados pela Emplasa (2011), que possui significativo potencial para a realização de mapeamentos morfológicos, geomorfológicos e geotécnicos, tendo em vista que são dados obtidos em escala de detalhe (1: 25.000) e que consiste no mais atual levantamento sistemático disponível para a abrangência de todo o território do estado de São Paulo.

O presente artigo teve como objetivo avaliar a viabilidade da utilização de recursos técnicos disponíveis nos softwares de SIG, conforme os procedimentos técnicos e metodológicos estabelecidos por Nepomuceno e Luchiari (2014), para a classificação morfológica semi-automatizada na escala de detalhe, escala 1:25.000, em bacias hidrográficas localizadas na Região do Pontal do Paranapanema, no estado de São Paulo, de acordo como quinto táxon da taxonomia do Relevo proposta por Ross (1992), referentes aos tipos e formas de vertentes, assim como a classificação das Feições Mínimas ou Unidades Elementares do Relevo, proposta por Colangelo (1996), utilizando como base de dados os MDE e ortofotos gerados no âmbito do projeto "Mapeia São Paulo", realizado pela Emplasa, que possuem precisão e acurácia compatíveis até a escala 1:25.000 (EMPLASA, 2011).

\section{2. Área de estudo}

As duas bacias hidrográficas analisadas se localizam na região do Pontal do Paranapanema, oeste paulista, sendo sub-bacias de quinta e quarta ordem, de acordo com a proposta de Strahler (1952), do rio Santo Anastácio, identificadas, respectivamente como I e II nesta pesquisa. O rio Santo Anastácio, segundo o relatório do Comitê da bacia hidrográfica do Pontal do Paranapanema, (DAEE. CBH-PP, 2016), é um dos grandes responsáveis pelo abastecimento de grande parte da região do Pontal.

O oeste paulista é caracterizado pelo clima Tropical, com inverno seco e verão úmido. Por estar contido em uma área de transição entre os sistemas inter e extratropical, em que há o choque das massas Tropical Atlântica, Tropical Continental e a massa Polar Atlântica (MONTEIRO, 1973).

A região, por sua vez, está contida na Bacia Sedimentar do Paraná, cujas formas presentes referem-se a planaltos e chapadas, formas típicas de bacias sedimentares com gênese entre o Devoniano e o Cretáceo, como afirma Penteado (1976). Ambas bacias hidrográficas selecionadas, estão inseridas na Morfoescultura do Planalto Ocidental Paulista, que apresenta um relevo suavemente ondulado, com a presença marcante de colinas extensas e baixas de topos aplanados (ROSS; MOROZ, 1997), mais especificamente na sub-unidade denominada Planalto Centro Ocidental de acordo com o Mapa Geomorfológico do Estado de São Paulo de autoria de Ross e Moroz, (1997). Este compartimento conta com um modelado de relevo caracterizado pelo predomínio de Padrões de Formas Semelhantes do tipo denudacionais com colinas amplas de topo convexos (Dc) e topos aplanados ou tabulares (Dt), caracterizados por altimetrias entre 300 e 700m (ROSS; MOROZ, 1997). 
A região conta com um recobrimento vegetacional de apenas $8 \%$ de sua extensão total, condizente a Floresta Estacional Semidecidual e Formação Arbórea-Arbustiva em Região de Várzeas. Algumas áreas de proteção estão inseridas no Pontal do Paranapanema, como Parque Estadual do Morro do Diabo, Estação Ecológica Mico Leão Preto, Reserva Particular de Patrimônio Natural (RPPN) Mosquito e RPPN Vista Bonita (DAEE/CBH-PP, 2016).

Contudo, segundo o comitê de bacia hidrográfica da UGRHI-22 (2016) há a preponderância de pastagens destinadas à criação de gado solto leiteiro e de corte, especialmente ao longo da bacia hidrográfica do Rio Santo Anastácio, o que pode ser observado nas sub bacias analisadas, sendo compostas, quase que totalmente, por pastagens.

No que se refere aos solos da região, observa-se a presença de duas formações condizentes ao Latossolo Vermelho-Amarelo e ao Podzólico vermelho-amarelo (atualmente denominado Argissolo vermelhoamarelo), com textura média a arenosa (ROSS; MOROZ, 1997). De acordo com Prado (1996) o primeiro tipo de solo citado apresenta pouca fertilidade em decorrência do seu baixo índice de bases trocáveis e de micronutrientes, sendo uma das razões pelas quais há uma limitação de uso. Já ao que se refere ao Argissolo Vermelho-Amarelo a Embrapa afirma que tal solo apresenta grande teor de ferro, e sendo encontrado em encostas com 8 a $20 \%$ de declive.

\section{Materiais e métodos}

Inspirando-se nas ideias de compartimentação das vertentes de autores como Hack e Goodlett (1960), Throeh (1965) e Ruhe (1975); Colangelo (1996) propôs a divisão das vertentes em nove formas elementares do relevo (feições mínimas) passíveis de serem cartografadas. Tais formas, segundo o autor, apresentam caráter de comportamento hídrico também, podendo ser delimitados comportamentos de concentração, dispersão ou transição, conforme se encontra representado na Tabela 1.

Tabela 1: Feições mínimas propostas por Colangelo (1996).

\begin{tabular}{ccccc}
\hline FM & Perfil & Planta & Fluxo Resultante & Domínio \\
\hline 1 & $\mathrm{CV}$ & $\mathrm{CV}$ & Hiperdispersor & Dispersor \\
2 & $\mathrm{CV}$ & $\mathrm{R}$ & Mesodispersor & Dispersor \\
& $\mathrm{L}$ & Longitudinal & \\
3 & $\mathrm{CV}$ & $\mathrm{CC}$ & Hipodispersor & Dispersor \\
4 & $\mathrm{R}$ & $\mathrm{CV}$ & Mesodispersor & Dispersor \\
& & & Radial & \\
5 & $\mathrm{R}$ & $\mathrm{R}$ & Transição ou Neutro & Transição \\
6 & $\mathrm{R}$ & $\mathrm{CC}$ & Mesoconcentrador & Concentrador \\
& $\mathrm{CC}$ & Radial & Concentrador \\
7 & $\mathrm{CC}$ & $\mathrm{R}$ & Hipoconcentrador & Concentrador \\
8 & $\mathrm{CC}$ & $\mathrm{CC}$ & Longitudinal & Concentrador \\
& & Hiperconcentrador & \\
\hline
\end{tabular}

$\mathrm{CV}=$ Convexo, $\mathrm{R}=$ Retilíneo, $\mathrm{CC}=$ Côncavo.

Já a segunda legenda utilizada neste artigo, elaborada por Ross (1992), corresponde a divisão do modelado terrestre em 6 táxons, relacionados a Morfoestrutura ( $1^{\circ}$ táxon), Morfoescultura $\left(2^{\circ}\right.$ táxon), Padrões de Formas semelhantes ( $3^{\circ}$ táxon), Tipos de Formas do relevo ( $4^{\circ}$ táxon) Tipos e Formas de Vertentes ( $5^{\circ}$ táxon) e Formas de Processos Atuais ( $6^{\circ}$ táxon). O presente artigo, já que tem como foco o mapeamento geomorfológico em escala de detalhe, atentou-se apenas ao quinto táxon estipulado pelo autor, o qual pode ser relacionado a unidade de vertente em si, bem como a divisões geométricas destas, atentando também as distintas dinâmicas intrínsecas a cada unidade.

Como já mencionado, para a realização de tais classificações nas bacias hidrográficas tomadas como objeto de pesquisa, foram utilizados como base de dados os Modelos Digitais de Elevação (MDEs) e ortofotos disponibilizados pela Emplasa (2011) através do projeto Mapeia São Paulo, na escala 1: 25.000.

Tal material foi processado em ambiente SIG, nos softwares Arcgis 9.3 da ESRI e QGIS 2.1.8, disponíveis no Laboratório de Aerofotogeografia Sensoriamento Remoto- Ailton Luchiari (LASERE), do departamento de Geografia da USP.

Florenzano (2008) destaca a possibilidade da utilização de técnicas de geoprocessamento para a geração de mapas morfológicos em escalas médias e grandes a partir do processamento de modelos digitais de 
elevação com o uso de SIG. Segundo a autora a utilização dessas técnicas permite a extração de diversas informações morfométricas, como a clinografia, hipsometria, curvatura em planta e em perfil, por exemplo.

Nesse mesmo sentido, Nepomuceno e Luchiari (2014) estabeleceram um conjunto de procedimentos técnicos e metodológicos de processamentos de MDE em ambiente SIG orientados para a classificação morfológica de detalhe a partir da combinação, ou correlacionamento, destes dados morfométricos, o qual apresentou significativa eficácia. Dessa forma esse conjunto de procedimentos foi adotado como referência técnica e operacional para a realização dessa pesquisa.

Para o processamento dos MDE foi necessário inicialmente a produção de um mosaico das áreas de análise. Posteriormente, foram aplicados filtros com o objetivo de evitar possíveis inconsistências hidrológicas e do terreno nos MDEs. A partir de então, foram extraídos dados morfométricos, além da cartografia de base, necessária para a posterior formulação da classificação morfológica, como mapas hipsométrico, clinométrico, orientação de vertente, rede de drenagem e curvaturas em planta e em perfil.

Para a classificação morfográfica, as curvaturas em planta e perfil das sub-bacias hidrográficas analisadas foram extraídas para a obtenção das classes de curvatura em: côncavo, retilíneo ou convexo, baseando-se no trabalho de Valeriano (2008), sendo, posteriormente, realizada a combinação dessas variáveis de maneira a permitir a classificação e delimitação morfológica, propriamente dita, conforme os procedimentos técnicos e metodológicos estabelecidos por Nepomuceno e Luchiari (2014).

Outro procedimento adotado foi a realização de trabalhos de campo nas sub-bacias hidrográficas estudadas, com o intuito de reconhecer e validar a divisão entre as classes de vertente extraídas durante o processo de classificação e mapeamento. Dessa forma, foi possível a elaboração de uma tabela (Tabela 2) com amostras do que fora observado em campo.

Tabela 2: Interpretação das feições.

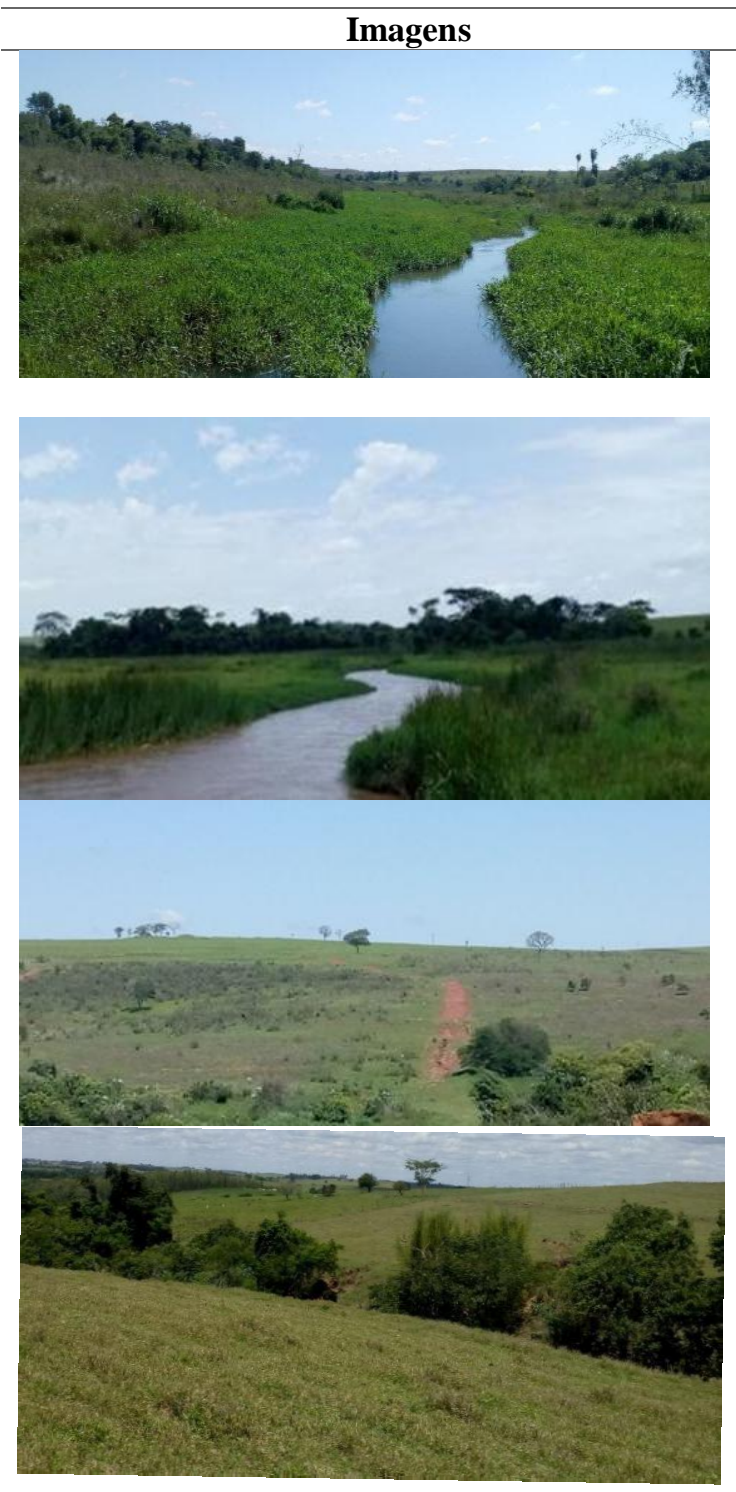
Canal Principal da bacia N e $21^{\circ} 57^{\prime} 50.8^{\prime \prime} \mathrm{S}$ respectiva planície de $51^{\circ} 57^{\prime} 27.9^{\prime \prime} \mathrm{W}$ inundação do rio Santo Anastácio.

Canal Fluvial e respectiva planície de inundação do compartimento geomorfológico Dc14

Topo de interflúvio convexo suave, de baixa declividade

$21^{\circ} 57^{\prime} 46.1^{\prime \prime S}$
Vertentes convexas suaves e vegetação ciliar localizada em vale em "V", encaixado $51^{\circ} 57^{\prime} 40.9^{\prime \prime} \mathrm{W}$

21 ${ }^{\circ} 59^{\prime} 27.9^{\prime \prime S}$

$51^{\circ} 58^{\prime} 30.3^{\prime \prime} \mathrm{W}$

$22^{\circ} 16^{\prime} 07.0^{\prime \prime} \mathrm{S}$ $51^{\circ} 26^{\prime} 37.3^{\prime \prime} \mathrm{W}$ 


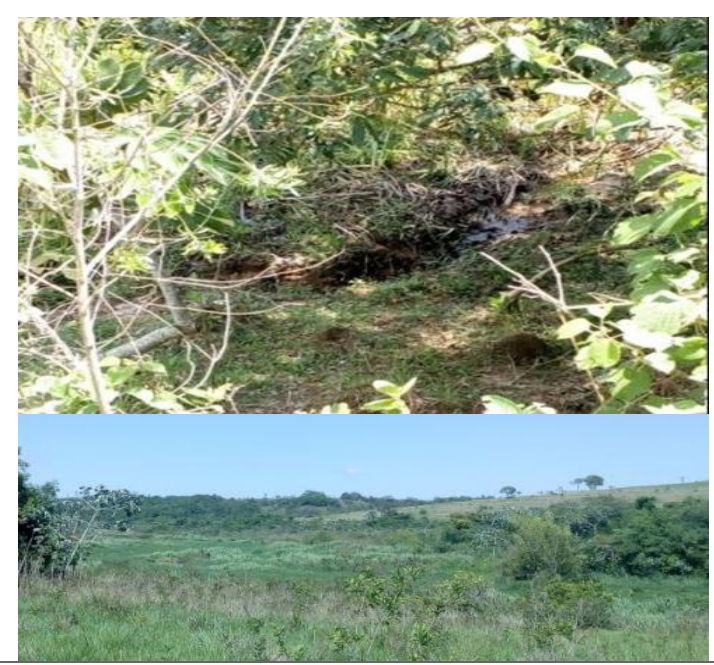

\begin{abstract}
Afloramento de nascente em $22^{\circ} 16^{\prime} 07.0^{\prime \prime} \mathrm{S}$ anfiteatro de cabeceira de $51^{\circ} 266^{\prime} 37.3^{\prime \prime} \mathrm{W}$ drenagem
\end{abstract}

Vertentes retilíneas e $21^{\circ} 57^{\prime} 39.5^{\prime \prime} \mathrm{S}$
convexas, com a presença $51^{\circ} 57^{\prime} 28.2^{\prime \prime} \mathrm{W}$
de vegetação de pequeno
porte.

Fonte: elaborados pelos autores

\title{
4. Resultados
}

No mapeamento guiado pela proposta de Colangelo (1996), foi possível extrair nove formas, na bacia hidrográfica de quinta ordem (I); e oito formas na bacia hidrográfica de quarta ordem (II), como pode ser observado na Tabela 3 e nas Figuras 1 e 2 seguir. Para tanto, foram interseccionadas as curvaturas em planta e perfil, gerando as nove formas elementares.

Com base na tabela produzida, podemos perceber que na bacia hidrográfica de quinta ordem I há um predomínio de feições mínimas correlacionadas a convexidade, apresentando ao todo 22,34 $\mathrm{Km}^{2}$, atreladas a um comportamento hídrico de fluxo dispersor, mais especificamente de Hiperdispersor (1), Mesodispersor longitudinal (2), hipodispersor (3) e mesodispersor radial (4).

Já a bacia hidrográfica de quarta ordem (II), não apresenta a feição mínima 3 (hiperdispersor), e a área de maior abrangência está atrelada ao domínio concentrador de fluxos hídricos, que pode ser considerada a soma das áreas das feições 6,7,8 e 9, correspondendo a 15,27 Km²; ao passo que o domínio dispersor, referente a soma das áreas das feições 1,2 e 4 apresenta somente $13,97 \mathrm{Km}^{2}$.

Para a aplicação da legenda proposta por Ross (1992) foram utilizados o mapa clinográfico, produzido a partir das classes de declividade propostas pela EMBRAPA (1979), em que são divididas em: plano (0-3\%), levemente ondulado (3-8\%), ondulado (8-20\%), fortemente ondulado (20-45\%), montanhoso (45-74\%) e fortemente montanhoso (>75\%); em conjunto com a intersecção já produzida das curvaturas em planta e perfil. A classificação partiu da combinação dessas variáveis morfométricas conforme apresentado na Tabela 4, o que possibilitou a compartimentação em quatro classes diferentes, sendo duas delas subdivididas de acordo com os valores médios de declividade, no caso das vertentes convexas e vertentes retilíneas.

A classificação do relevo de acordo com o quinto táxon proposto por Ross (1992), foi realizada a partir da extração dos tipos de vertentes das bacias hidrográficas analisadas, distinguindo-se as formas convexas, retilíneas e côncavas. Posteriormente foram correlacionadas as variáveis morfométricas de curvatura e declividade com o objetivo de diferenciação de classes de tipos de vertentes. Dessa maneira, nas sub-bacias analisadas foram encontradas quatro unidades morfológicas, e mais 5 subunidades, conforme explicitados na Tabela 4.

Tabela 3: Feições mínimas do relevo das bacias hidrográficas analisadas

\begin{tabular}{ccc}
\hline Classes de feições mínimas & Sub-bacia I $\left(\right.$ área em $\left.\mathbf{~ k m}^{2}\right)$ & Sub-bacia II $\left(\right.$ área $\left.\mathbf{~ e m ~} \mathbf{~ k m}^{\mathbf{2}}\right)$ \\
\hline 1 & 12,9 & 10,92 \\
2 & 4,12 & 1,32 \\
3 & 1,16 & $-1,32$ \\
4 & 4,16 & 1,73 \\
5 & 5,08 & 1,24 \\
6 & 3,63 & 1,35 \\
7 & 1,08 & 0,93 \\
8 & 3,94 & 1,69 \\
9 & 13,32 & 11,30 \\
\hline
\end{tabular}




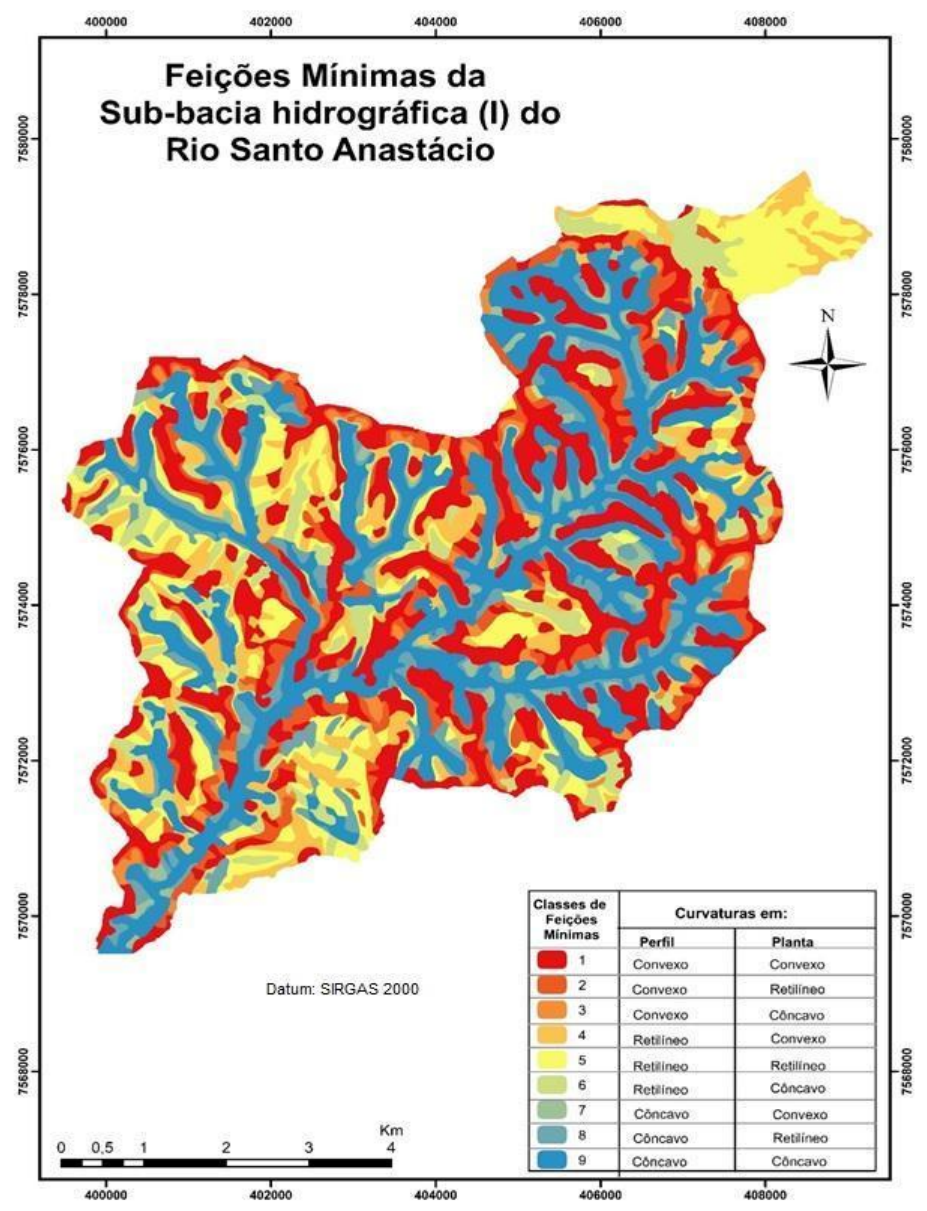

Figura 1: Feições mínimas da sub-bacia hidrográfica I do Rio Santo Anastácio. Fonte: elaborados pelos autores.

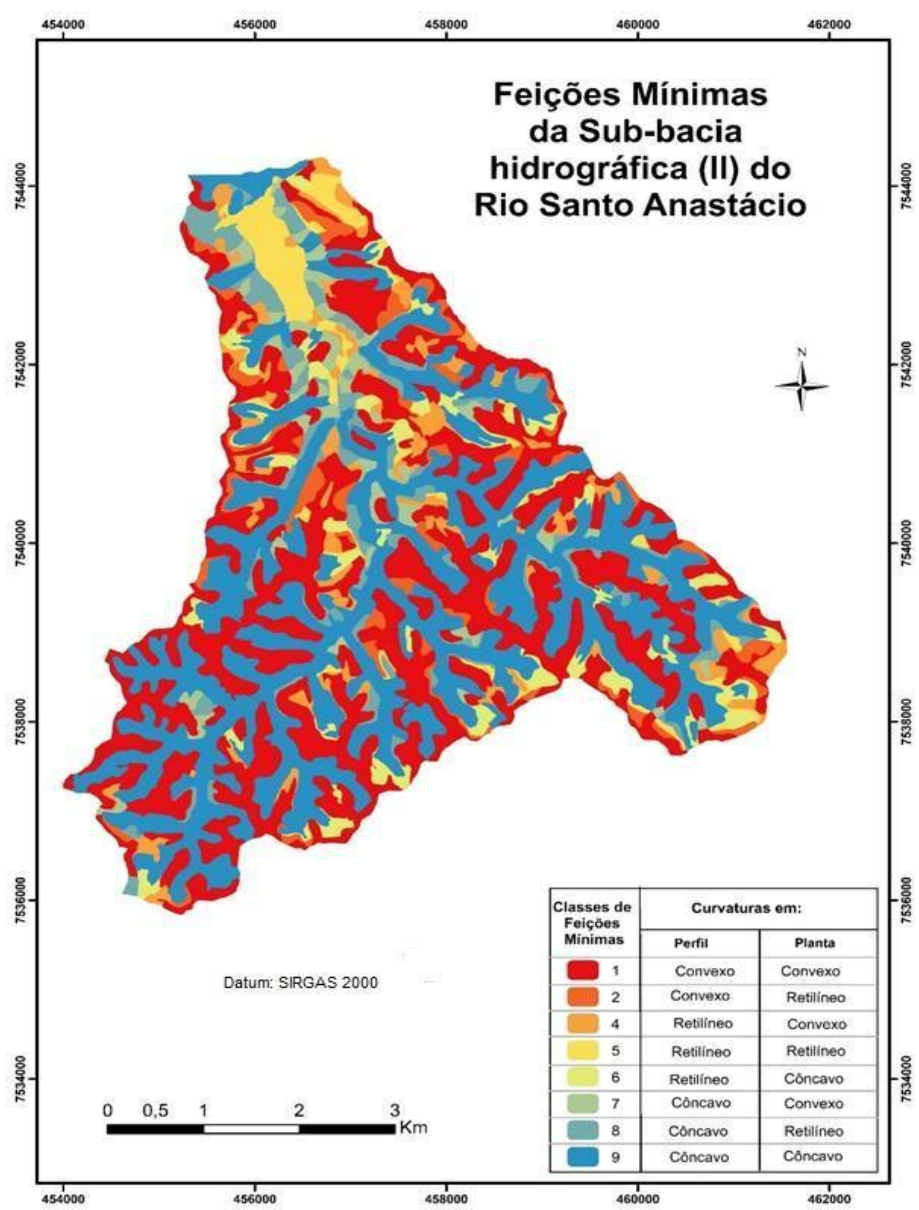

Figura 2: Feições mínimas da sub-bacia hidrográfica II do rio Santo Anastácio. Fonte: elaborados pelos autores. 
Tabela 4: Classificação dos Tipos de Vertentes e respectivas áreas de abrangência.

\begin{tabular}{lcc}
\hline \multicolumn{1}{c}{ Tipos de Vertentes } & Sub-bacia I (área em km²) & Sub-bacia II (área em km $\left.\mathbf{~ k m}^{\mathbf{2}}\right)$ \\
\hline Topo de interflúvio & 6,87 & 1,19 \\
Vertentes convexas de baixa declividade (0-3\%) & 0,52 & 0,75 \\
Vertentes convexas de média declividade (3-8\%) & 10,30 & 11,42 \\
Vertentes convexas de alta declividade (8-20\%) & 4,82 & 0,41 \\
Vertentes retilíneas de baixa declividade (0-3\%) & 1,61 & 0,99 \\
Vertentes retilíneas de média declividade (3-8\%) & 7,92 & 1,80 \\
Vertentes côncavas e vales & 18,53 & 13,83 \\
\hline
\end{tabular}

Fonte: elaborados pelos autores

Dessa forma, podemos observar que as classes referentes às vertentes convexas de média declividade, e às vertentes côncavas se mostram dominantes em ambas bacias hidrográficas estudadas. Já as classes de menor predominância são os topos de interflúvio, com $6,87 \mathrm{Km}^{2}$ e $1,19 \mathrm{Km}^{2}$, e as vertentes convexas de baixa declividade, com $0,52 \mathrm{Km}^{2}$ e $0,75 \mathrm{Km}^{2}$, além das vertentes convexas de alta declividade, apresentando apenas $0,41 \mathrm{Km}^{2}$, no caso da bacia II. As distribuições dessas formas nas duas bacias analisadas estão representadas nas Figuras 3 e 4 .

Através dos resultados obtidos a partir dos procedimentos técnicos laboratoriais em conjunto com o trabalho de campo realizado, podemos afirmar que as bacias apresentam um relevo predominantemente suave, de pouca dissecação, e baixa amplitude altimétrica. Pode se afirmar, também, que as áreas de estudo apresentam intensa presença de vertentes convexas de baixa a média declividade (0-8\%), e Feições Mínimas de comportamento hídrico dispersor.

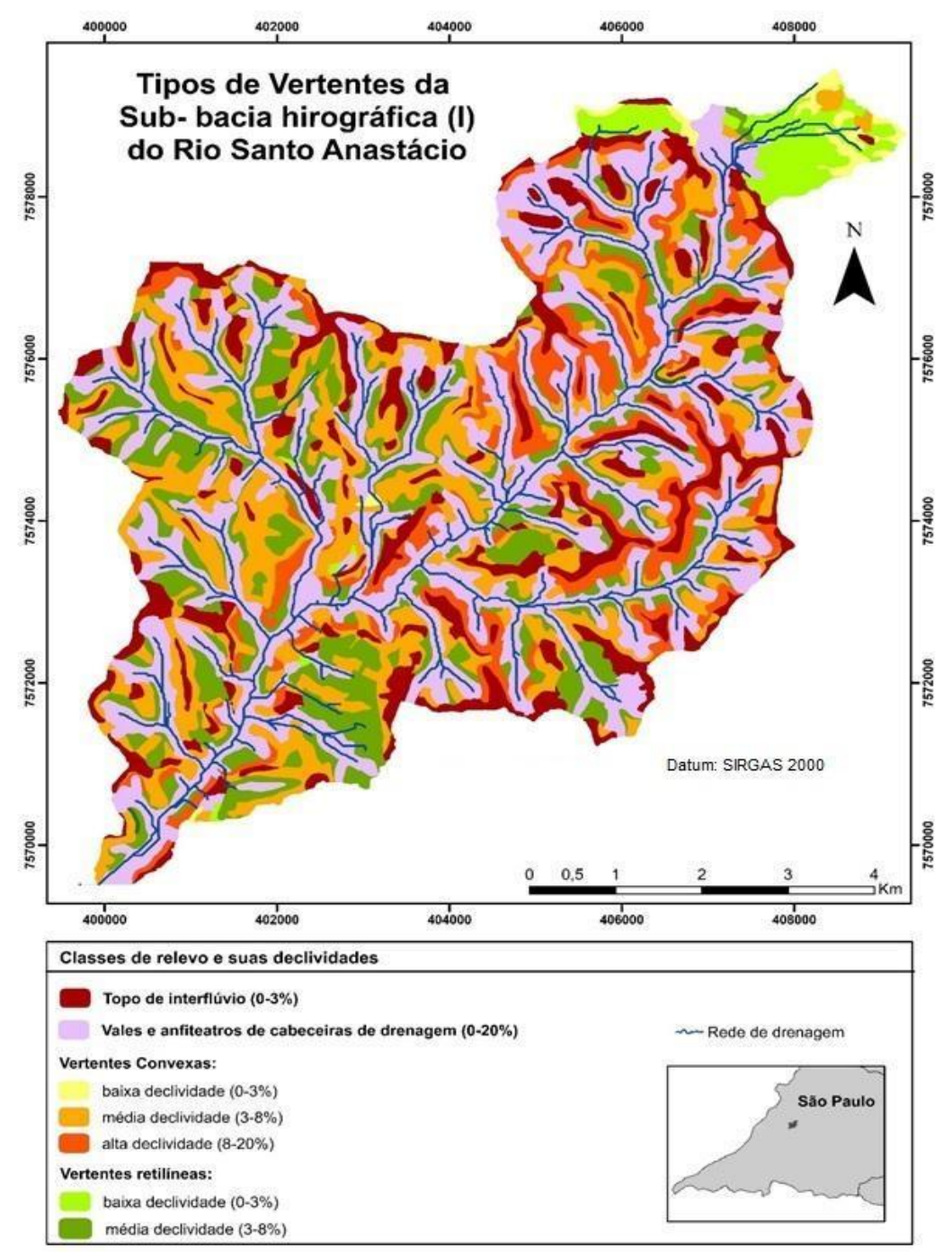

Figura 3: Tipos de vertentes da Sub-bacia hidrográfica I do Rio Santo Anastácio. Fonte: elaborados pelos autores. 


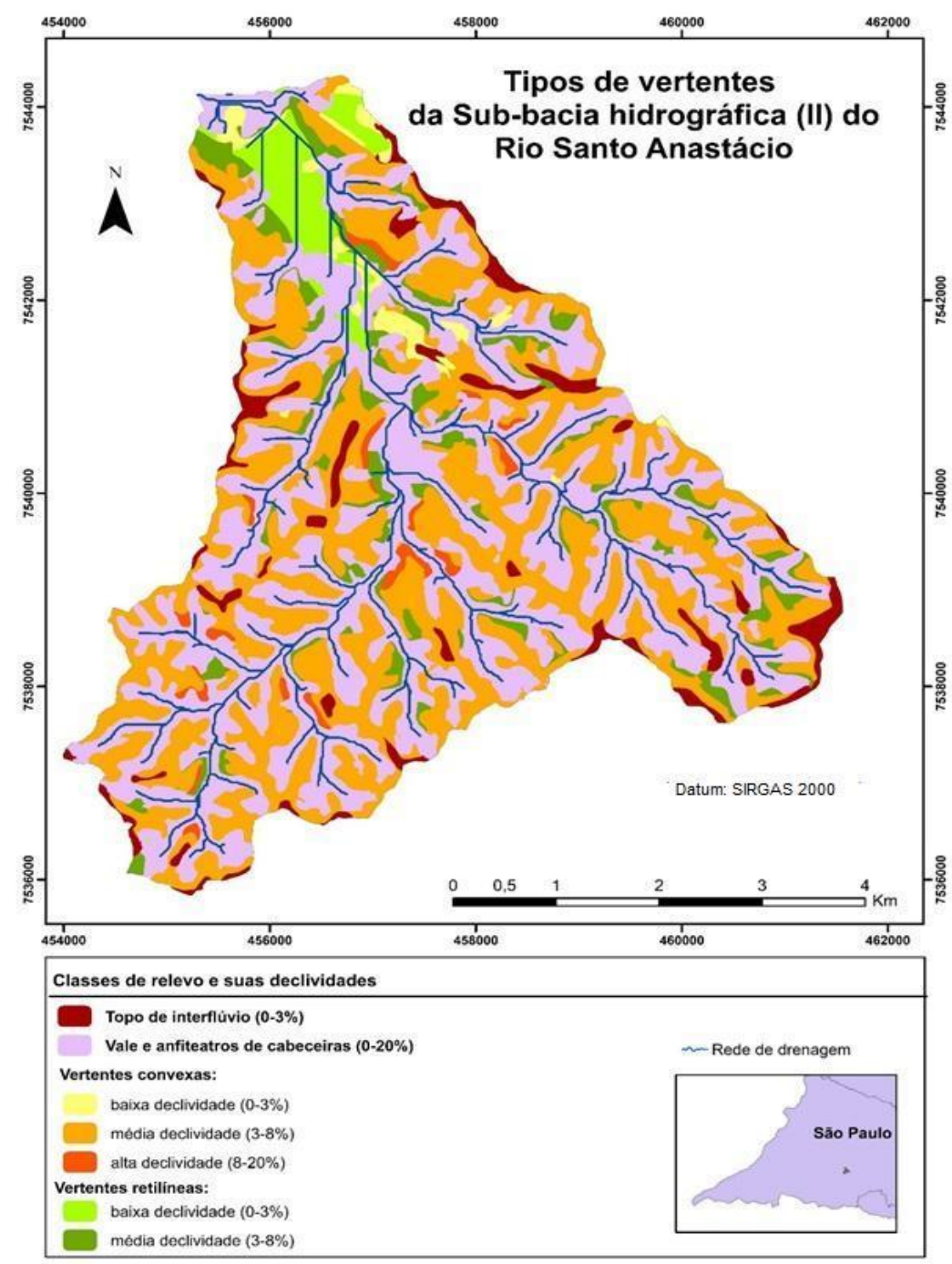

Figura 4: Tipos de vertentes da Sub-bacia hidrográfica II do Rio Santo Anastácio. Fonte: elaborados pelos autores

O uso da terra destinado a criação de gado solto, aliado a características físicas da região, como tipo de solo e regime de chuvas, acarretaram no aparecimento de formas relacionadas com ravinas e voçorocas na região. Desse modo, analisando em conjunto com a presença de formas côncavas, tais processos apresentam uma grande ameaça de perda de solo e em uma futura incapacidade de utilização de tais áreas. Tais constatações apontam para a possibilidade de incorporação das variáveis morfométricas utilizadas nessa pesquisa e mesmo das classificações morfológicas obtidas, em modelos que visam, diagnosticar, quantificar e/ou prevenir a ocorrência de processos erosivos tanto em ambiente naturais, quanto antropizados, como os modelos de aptidão agrícola, de capacidade de uso da terra, de diagnóstico de fragilidade ambiental, entre outros.

\section{Conclusões}

As técnicas empregadas para a geração dos Mapas da Figuras 1 e 2 de "Feições Mínimas" ou "Unidades Elementares do Relevo", de maneira semelhante às encontradas por Nepomuceno e Luchiari (2014) para o caso do Município de Salesópolis, se mostraram eficientes e adequadas, tendo em vista que são, basicamente, a combinação entre as informações referentes a curvatura em planta e em perfil, dados que são facilmente obtidos de forma automática na maioria de softwares de SIG disponíveis atualmente. Tal fato proporciona uma aplicação mais simples e eficiente no tempo de execução quando comparado com as técnicas convencionais baseadas na fotointerpretação. Além disso, o cálculo automatizado destas variáveis elimina por completo a subjetividade no processo, pois fornecem os valores absolutos de variações das curvaturas nas projeções em planta e em perfil, o que permite a delimitação precisa da área de abrangência de cada feição identificada, bem como a classificação completa das áreas objetos de pesquisa, constando todas as feições que nela ocorrem. 
Os Mapas das Figuras 3 e 4, por sua vez, consistem em produtos do correlacionamento entre as classes de todas as variáveis morfométricas geradas, conforme explicitado na Tabela 4. Dessa maneira procedimento visa simular alguns dos critérios utilizados pelo pesquisador durante a fotointerpretação, utilizando-se, no entanto, de combinações entre variáveis morfométricas (curvatura em planta, curvatura em perfil e clinografia), na finalidade de identificar homogeneidades no relevo, o que consiste a sua compartimentação morfológica.

Pode-se concluir que tais combinações são similares às relações que o fotointérprete tem que definir para realizar a restituição morfológica sobre fotografias aéreas verticais com o uso de estereoscópios, como, por exemplo: na identificação de variações clinográficas abruptas e suaves indicando, muitas vezes, os limites das planícies e dos topos de interflúvios com as vertentes; ou na identificação de formas côncavas em planta e em perfil nas vertentes, que representam morfometricamente tanto os vales quanto os anfiteatros formadores de canais fluviais de $1^{\circ}$ ordem;ou ainda, as formas convexas em perfil e em planta, com baixas declividades, traduzem topos de interflúvios; e assim sucessivamente.

Pode se dizer, portanto, que o estabelecimento de limiares de classes nas variáveis morfométricas, bem como as combinações propostas, consistem em procedimentos que pretendem, ao menos parcialmente, simular alguns dos critérios utilizados na fotointerpretação para o estabelecimento das mesmas categorias, podendo ser entendido, portanto, como uma "classificação orientada aos objetos", no caso: dos compartimentos morfológicos de $5^{\circ}$ táxon, a partir da combinação entre classes de variáveis morfométricas. Dessa maneira, pode-se concluir que a utilização destes procedimentos técnicos e metodológicos também se mostraram consideravelmente úteis e eficientes, pois torna o processo mais rápido além de atribuir maior objetividade a análise.

Conclui-se, portanto, que os procedimentos técnicos e metodológicos estabelecidos por Nepomuceno e Luchiari (2014) para a classificação morfológica na escala de detalhe também podem ser aplicados sobre os dados provenientes do projeto Mapeia São Paulo da Emplasa (2011) e para outras regiões do estado de São Paulo, como a Região do Pontal do Paranapanema.

\section{Agradecimentos}

Os autores agradecem a assistência técnica do Laboratório de Aerofotogeografia Sensoriamento Remoto- Ailton Luchiari (LASERE) do Departamento de Geografia da USP. Além do apoio e o financiamento da Fundação de Amparo à Pesquisa do Estado de São Paulo (FAPESP), crucial para a realização deste estudo.

\section{Referências}

COLÂNGElO, A. C. Modelo de Feições Mínimas ou das Unidades Elementares do Relevo: um suporte cartográfico para mapeamentos geoecológicos. In: Revista do Departamento de Geografia, São Paulo, n.10, p.29-40, 1996.DOI:RDG.1996.0010.0003.

DAEE. CBH-PP. Relatório de Situação dos Recursos Hídricos do Pontal do Paranapanema. Ano base 2016. Disponivel em:<http://cbhpp.org/publicacoes-2/>

EMPRESA BRASILEIRA DE PESQUISA AGROPECUÁRIA - EMBRAPA. Serviço Nacional de Levantamento e Conservação de Solos. Rio de Janeiro, RJ. Súmula da X Reunião Técnica de Levantamento de Solos. Rio de Janeiro, p.83, 1979.

EMPRESA BRASILEIRA DE PESQUISA AGROPECUÁRIA - EMBRAPA. Disponível em: http://www.agencia.cnptia.embrapa.br/Agencia16/AG01/arvore/AG01_97_10112005101957. Acesso em: Mar. 2019.

FlorenZANO, T. G (Org). Geomorfologia: Conceitos e tecnologias atuais. São Paulo: Oficina de Textos, 2008.

HACK,J. J.; GOODLETT, J. C. Geomorphology and Forest Ecology of a Mountain Region in the Central Appalachians, United States Geological Survey Prof Paper, p.347-366. 1960. DOI: 10.3133/pp347. 
MONTEIRO, C. A. de F. A Dinâmica Climática e as Chuvas no Estado de São Paulo - estudo geográfico sob forma de atlas. São Paulo: Universidade de São Paulo/ Instituto de Geografia, p.129,1973.

NEPOMUCENO, P. L. M.; LUCHIARI, A. Mapeamento Morfológico de Detalhe: Experiências para a integração entre técnicas de Fotointerpretação e Sistemas de Informações Geográficas no Município de Salesópolis-SP. Revista do Departamento de Geografia, São Paulo, n.28, p.198-220, 2015. DOI: $10.11606 /$ rdg.v28i0.530

NEPOMUCENO, P. L. M. Geoprocessamento nos mapeamentos de inventário dos Planos de Manejo de Unidades de Conservação paulistas: avaliação dos casos do PETAR e da APA-VRT a partir da revisão integrada da literatura. São Paulo: Faculdade de Filosofia, Letras e Ciências Humanas, Universidade de São Paulo, 2016. Tese de Doutorado em Geografia Física. Disponível em: <http://www.teses.usp.br/teses/disponiveis/8/8135/tde-12092016-121146/>.

PENTEADO, M. M. Fundamentos de Geomorfologia. Rio de Janeiro: Fund. IBGE, 1976.

PRADO, H. Manual de Classificação de Solos do Brasil. 3a ed. UNESP. 1996.

ROSS, J. L. S. Registro cartográfico dos fatos geomórficos e a questão da taxonomia do relevo In: Revista do Departamento de Geografia, São Paulo, n.6, p.17-29, 1992. https://doi.org/10.7154/RDG.1992.0006.0002

ROSS, J. L. S.; MOROZ, I. C. Mapa Geomorfológico do Estado de São Paulo (1:500.000) - Memorial Explicativo vol. 1. São Paulo-SP. FFLCH/USP, IPT,FAPESP, 1996.

RUEH, R. V. Geomorphology: Geomorphic Processes and Surficial Geology. Houghten Miflin Company, p.246, 1975.

STRAHLER, A.N. Hypsometric (area-altitude) - analysis of erosion al topography. Geol. Soc. America Bulletin. n. 63, p. 1117-1142, 1952.

TROEH, F. R. Landform Equations Fitted to Countour Maps, American Journal of Sciences, 263 p. 1965.DOI: $10.2475 /$ ajs.263.7.616.

VALERIANO, M.M. Dados Topográficos. In: Geomorfologia: conceitos e tecnologias atuais. São Paulo: Oficina de Textos, 2008. 